\title{
Malakoplakia of the Urinary Bladder and Unilateral Ureter
}

\author{
Saddaf Hina1', Aisha Hasan², Nadeem Iqbal2, Muhammad Usman Shabbir² and Abdul Ahad Ehsan Sheikh²
}

\begin{abstract}
Malakoplakia is an unusual acquired granulomatous disease that can affect many systems including urogenital tract. It presents a huge diagnostic challenge as it can mimic malignancy. We report a 55-year diabetic woman who presented with history of macroscopic hematuria and right flank pain. On investigations, ultrasound of kidney, ureter and bladder (KUB) showed right hydronephroureter, and CT KUB showed right moderate hydronephroureter and right ureteric stone. Endoscopic examination revealed multiple white plaques involving urinary bladder and right ureter. The diagnosis of malakoplakia was based on microscopic findings that are specific for its diagnosis.
\end{abstract}

Key Words: Malakoplakia, Urinary bladder, Malignancy.

\section{INTRODUCTION}

Malakoplakia is defined histopathologically by MichaelisGutmann bodies; whilst the exact etiology is unknown, it is believed to be associated with defective macrophage function. ${ }^{1,2}$ Malakoplakia is very rare granulomatous disease of infective etiology and can present as nodules, plaques or ulcers. The clinical presentation of malakoplakia varies widely, especially in the urinary bladder, where it can mimic bladder tumor. 3 Malakoplakia typically involves the urinary tract, commonly the bladder $(40 \%)$, renal parenchyma $(16 \%)$, and rarely the ureter $(11 \%)$. However, it can affect any organ of the body. ${ }^{4}$ The disease usually affects immunocompromised individuals. ${ }^{5}$ The clinical diagnosis of malakoplakia is very difficult and requires confirmation by histopathology.

We present a 55-year woman who had malakoplakia diagnosed after biopsy of bladder and ureteric lesion. To the best of the authors' knowledge, this is the first reported case of malakoplakia involving the bladder and unilateral ureter in Pakistan.

\section{CASE REPORT}

A 55-year female presented to the urology outpatient clinic with complaints of frequency, urgency, dysuria, gross hematuria with passage of clots and right flank pain for one month. Patient also had history of recurrent urinary tract infections (UTI). She was known diabetic and was on oral hypoglycemics for the last 16 years.

On clinical examination, body temperature was $36.5^{\circ} \mathrm{C}$, blood pressure $130 / 82 \mathrm{mmHg}$, pulse rate 80 beats per

1 Department of Urology, PIMS, Islamabad, Pakistan

2 Department of Urology, Shifa College of Medicine, Shifa International Hospital, Islamabad, Pakistan

Correspondence: Dr. Nadeem Iqbal, Department of Urology,

Pakistan Kidney Institute, Shifa International Hospital,

Sector H-8/4, Islamabad, Pakistan

E-mail:dr_nadeemiqbal84@yahoo.com

Received: May 23, 2018; Accepted: November 12, 2018 minute, and respiratory rate 14 breaths per minute. Laboratory investigations showed hemoglobin of $12.2 \mathrm{~g} / \mathrm{dl}$; serum urea of $6.2 \mathrm{mmol} / \mathrm{l}$; and serum creatinine 100 umol/l $(1.13 \mathrm{mg} / \mathrm{dl})$. Midstream urine showed $\mathrm{pH}$ of 5 , numerous red blood cells (RBCs) and numerous leucocytes. Urine culture revealed E. coli, sensitive to quinolones. Renal ultrasonography showed moderate right hydronephrosis and proximal right hydroureter. Abdominal computed tomography (CT) showed right hydronephrosis and right hydroureter (Figure 1a) with a faintly visualised hyperdense focus at right vesicoureteric junction (VUJ), suggestive of a calculus or an encrusted lesion (Figure 1b).

On endoscopic examination, cystoscopy showed multiple nodular brush-like lesions with easy bleeding in the bladder, especially around right VUJ (Figure 2a). Biopsies of bladder were taken (Figure $2 \mathrm{~b}$ ) and lesions were fulgurated with holmium laser (Figure 3b). Right ureterorenoscopy done after resecting lesion around right VUJ, which revealed multiple white plaques involving whole right ureter (Figure 3a). Biopsies of ureteric lesions were taken. Microscopy revealed submucosa infiltrated by sheets of macrophages having

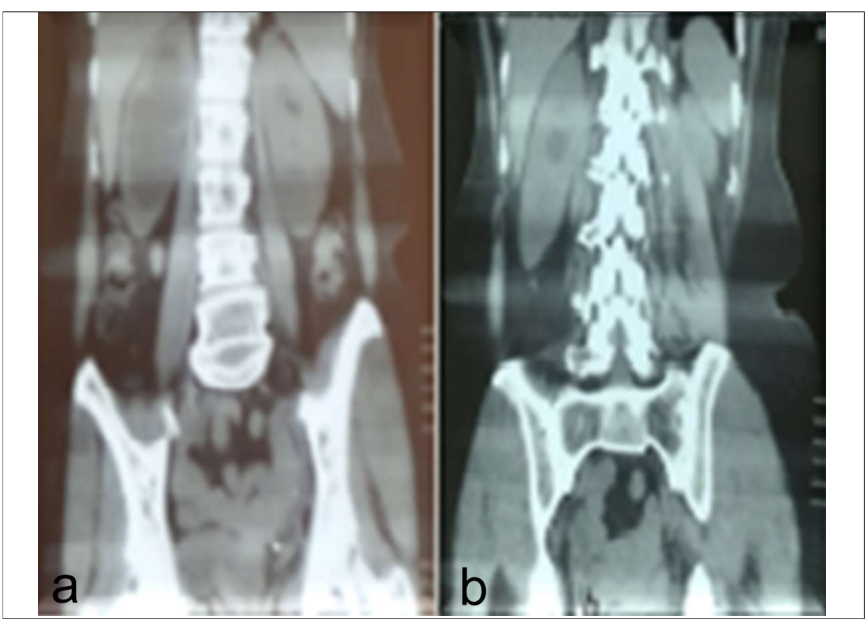

Figure 1: CT scan showing right hydronephroureter; and arrow pointing stone or encrusted lesion in distal ureter. 


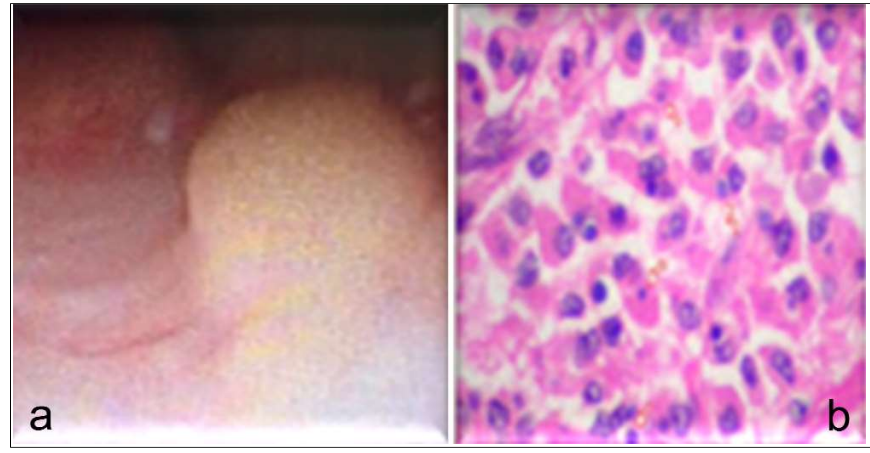

Figure 2: (a) Multiple nodular lesions in bladder near right ureteric orifice (vesicoureteric junction). (b) Arrow showing Michaelis-Gutmann bodies.

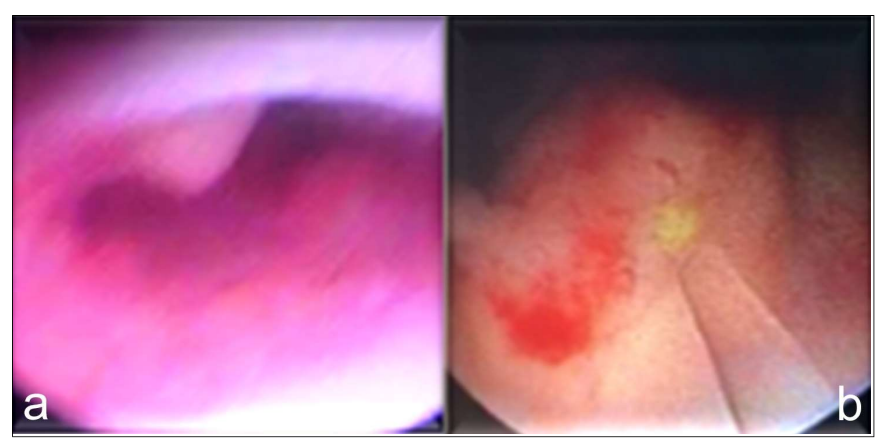

Figure 3: (a) Multiple nodular lesions in right distal ureter. (b) Right ureteric lesion fulguration done with holmium laser.

granular cytoplasm containing Michaelis-Gutmann bodies. Definitive diagnosis of malakoplakia was made. The patient was discharged on low dose antibiotics and ascorbic acid. Strict monthly follow-up was advised.

\section{DISCUSSION}

The first case of malakoplakia was described by von Hansemann in 1901 and in 1902 by Michaelis and Gutmann. The word malakoplakia was coined by von Hansemann in 1903 and is derived from the Greek words malakos (soft) and plakos (plaques), describing its usual clinical presentation as friable yellow soft plaques. ${ }^{4}$ It is characterised histologically by von Hansemann histiocytes and Michaelis Gutmann bodies. ${ }^{2,4}$ Von Hansemann cells are oval histiocytes which contain intracytoplasmic bodies called Michaelis Gutmann bodies. They have unique staining characteristics, being gram-negative and positive for alizarin red and von Kossa stain (calcium), Perls' stain and Prussian blue (iron), and Periodic Acid-Schiff stains. It may be incidentally diagnosed at biopsy as it may coexist with other chronic illnesses.1,4 Malakoplakia most commonly occurs in urogenital tract, but can involve gastrointestinal tract, mesenteric lymph nodes and retroperitoneum, bone, lungs, skin and brain. 4 Malakoplakia is four times more common in women and usually affects men above the age of 50 years. ${ }^{4}$

Malakoplakia presents clinically with wide spectrum of findings depending on organs involved. Bladder malakoplakia presents with irritative lower urinary tract symptoms (LUTS) and hematuria, mimicking malignancy. Ureteral malakoplakia may present with obstruction and formation of stricture. Renal parenchymal malakoplakia can cause fever, flank pain and mass.4,6 Testicular malakoplakia can manifest as epididmo-orchitis. Prostatic malakomlakia may mimic caricnoma of prostate as it may present as hard induration on digital rectal examination (DRE).5,6

The exact etiology of malakoplakia is uncertain, occurring commonly in immunocompromised individuals; and $E$. coli is the commonest pathogen associated with malakoplakia. ${ }^{7}$ Malakoplakia is believed to result from defective phagocytosis resulting in inadequate killing of bacteria due to deficiency of 3 to 5 guanosine monophosphate dehydrogenase. ${ }^{7}$ Decreased intracellular cyclic guanosine monophosphate (cGMP) levels may interfere with adequate microtubular function and lysosomal activity, leading to defective phagolysosomal activity. Inadequate killing of bacteria $(E$. coli) results in granulomatous reaction due to an accumulation of bacterial degradation products. ${ }^{7}$ However, partially digested bacteria become calcified gradually and accumulate in macrophages, forming the pathognomonic calcified intracellular inclusions called Michaelis-Gutmann bodies.3,7

Intraluminal protrusion of bladder mucosa occurs due to accumulation of macrophages in the lamina propria of urinary bladder, as in this case. Since this entity is rare and presents with gross hematuria, it can easily be misdiagnosed as malignancy.

Intravenous urography (IVU) is an important investigation in upper urinary tract malakoplakia. It can demonstrate hydronephroureter and multiple filling defects.1,7 However, we performed CT KUB instead of IVU, which showed hydronephroureter and ureteric stone/encrusted lesion in distal ureter.

Treatment of malakoplakia is mostly medical while surgical intervention may be required at times. There are no widely established guidelines for the medical treatment of malakoplakia, but most approaches involve antibiotics which concentrate intracellularly in macrophages such as quinolones (mainstay of treatment), trimethoprim and rifampicin. These can be used for prolonged period at low doses to prevent recurrence. Antibiotic therapy directed against E.coli in combination with surgery provides better chance of cure. Bethanecol, a choline agonist and ascorbic acid, may correct the decreased cGMP levels in monocytes (that interferes with complete bacterial killing). It may be used in combination with antibiotics. Moreover, as malakoplakia is associated with immunosuppression, discontinuation of immunosuppressive drugs is usually needed depending on the risk to benefit ratio.2,6,7

In conclusion, a high index of suspicion is required for the correct diagnosis and proper management of malakoplakia. 


\section{REFERENCES}

1. Shah V, Rupani A, Pathak HR. Malacoplakia of urinary bladder. Bom Hosp J 2010; 52:144-5.

2. Dong H, Dawes S, Philip J, Chaudhri S, Subramonian K. Malakoplakia of the urogenital tract. Urol Case Rep 2015; 3:6-8.

3. Ristic-Petrovic A, Stojnev S, Jankovic-Velickovic L, Marjanovic G. Malakoplakia mimics urinary bladder cancer: A case report. Vojnosanit Pregl 2013; 70:606-8.

4. Chen YC, Kuo HC. Malakoplakia of ipsilateral kidney, ureter and bladder. Tzu Chi Med J 2010; 22:103-5.
5. Patnayak R, Reddy MK, Subramanian S, Jena A, Ravisankar G, Dandu RS. An unusual case of bilateral hydroureteronephrosis caused by uretero-vesico malakoplakia in a youngmale: A case report and review of the literature. Cases J 2009; 2:7527.

6. Stanton MJ, Waxted W. Malacoplakia: A study of the literature and current concepts of pathogenesis, diagnosis and treatment. J Urol 1981; 125:139-46.

7. Ali Z, Farouk K, Khan MA, Hanif S, Javed J. Malakoplakia of the urinary bladder: A case report. J Postgrad Med Ins 2010; 24:165-7.

....น.... 